\title{
Comparative DFT Study of Phytochemical Constituents of the Fruits of Cucumis trigonus Roxb. and Cucumis sativus Linn.
}

\author{
Subarayan Bothi Gopalakrishnan,, Thangaraj Kalaiarasi, $^{2}$ and Ramasamy Subramanian ${ }^{3}$ \\ ${ }^{1}$ Department of Chemistry, Noorul Islam University, Kumaracoil, Kanyakumari, Tamil Nadu 629 180, India \\ ${ }^{2}$ Department of Pharmaceutical Chemistry, Manonmaniam Sundaranar University, Tirunelveli, Tamil Nadu 627152, India \\ ${ }^{3}$ Centre for Scientific and Applied Research, School of Basic Engineering and Sciences, PSN College of Engineering and Technology, \\ Tirunelveli, Tamil Nadu 627012, India
}

Correspondence should be addressed to Subarayan Bothi Gopalakrishnan; sgkmsu@yahoo.co.in

Received 12 June 2014; Revised 17 July 2014; Accepted 31 July 2014; Published 12 August 2014

Academic Editor: Xavier Ferrieres

Copyright (C) 2014 Subarayan Bothi Gopalakrishnan et al. This is an open access article distributed under the Creative Commons Attribution License, which permits unrestricted use, distribution, and reproduction in any medium, provided the original work is properly cited.

\begin{abstract}
The hepatoprotective active phytochemical constituents from the ethanolic extracts of the fruits of Cucumis trigonus Roxb. and Cucumis sativus Linn. were identified by GC-MS analysis. The density functional theory (DFT) of these molecules was calculated by density functional B3LYP methods using B3LYP/6-311++G(d,p) basis set. The optimized geometries of phytochemical constituents were evaluated. Physicochemical properties such as HOMO, LUMO, ionization potential, electron affinity, electronegativity, electrochemical potential, hardness, softness, electrophilicity, total energy, and dipole moment have also been recorded. These are very important parameters to understand the chemical reactivity and biological activity of the phytochemical constituents. Glycodeoxycholic acid and 2-(2-methylcyclohexylidene)-hydrazinecarboxamide were found to be effective drugs selected on the basis of their HOMO and LUMO energy gap and softness. The effective properties of these compounds may be due to the presence of amino, carbonyl, and alcohol as a functional group.
\end{abstract}

\section{Introduction}

DFT is the connection between the density and the wave function which was developed by Hohenberg and Kohn and stated in the Hohenberg-Kohn theorems [1, 2]. The first theorem shows that the Hamiltonian is fully determined by the ground state density, which means that all the physical properties of the system are completely determined by the ground state density. The second theorem demonstrates that the energy functional can be used to find the ground state density. Importantly, the excited states need to be determined in a different way. During the last decade, computer simulations based on a quantum-mechanical description of the interaction between electrons and atomic nuclei have strongly influenced the development of material science [3]. The major advantage from a computational point of view is that the electronic density depends only on the three variables of space, while the wave function for an $N$ electron system depends on $3 N$ variables.
DFT uses the B3LYP/6-311++G(d,p) hybrid functional in the gas phase. The presence of phytochemical constituents in the ethanolic extracts of the fruits of Cucumis trigonus and Cucumis sativus is responsible for curing liver disorder. Silymarin is used as a potential hepatoprotective agent. In the present work, a comparative DFT study of phytochemical constituents identified from the ethanolic extracts of the fruits of Cucumis trigonus and Cucumis sativus has been performed. Gaussian 09W program and Gauss-View molecular visualization program package on the personal computer have been used.

\section{Material and Methods}

2.1. Collection of Plant Materials. The fruits of Cucumis trigonus and Cucumis sativus were collected in the month of March and July, respectively, from Alangulam, Tirunelveli District, Tamil Nadu, and identified by Professor 
P. Jayaraman, Plant Anatomy Research Centre, West Tambaram, Chennai, Tamil Nadu, India (Registration Numbers of the Authentification Certificate: PARC/2013/2048 and PARC/2013/2047).

The voucher specimens (MSU/PHAR/HER-140 and MSU/PHAR/HER-141) have been preserved in the Herbarium of the Department of Pharmaceutical Chemistry, Manonmaniam Sundaranar University, Tirunelveli, Tamil Nadu, India.

2.2. Identification of Photochemical Constituents. Nine phytochemical constituents, namely, demeclocycline, glycodeoxycholic acid, $3 \alpha, 7 \alpha, 12 \alpha$-trihydroxycoprostanic acid, chlortetracycline, azafrin methyl ester, giganteumgenin $\mathrm{N}$, phorbol 12,13-dihexanoate, astaxanthin, and tetrahydrospirilloxanthin, were identified from the ethanolic extract of the fruits of Cucumis trigonus. Six phytochemical constituents, 2,3dihydro-3,5-dihydroxy-6-methyl-4H-pyran-4-one, 5-(hydroxymethyl)-2-furancarboxylic aldehyde, 4-hydroxy-3-methyl-2-butenyl-acetate, 2-(2-methylcyclohexylidene)-hydrazide carboxamide, n-hexadecanoic acid, and 1,2-benzenedicarboxylic acid-diisooctyl ester, were identified from the ethanolic extract of the fruits of Cucumis sativus using GC-MS $[4,5]$ analysis.

2.3. Computational Methods. All calculations were performed using Gaussian 09 software package [6], at the B3LYP/6-311++G(d,p) level of theory. Transitions to the lowest excited singlet electronic states of phytochemical constituents from the ethanolic extracts of the fruits of Cucumis trigonus and Cucumis sativus were computed by using the gradient corrected DFT with the three-parameter hybrid functional Becke3 (B3) for the exchange part and the Lee-Yang-Parr (LYP) correlation function, and HOMOLUMO energy level calculations have been carried out in the present investigation, using 6-311++G(d,p) Basis set with Gaussian 09W program package [7-9].

The chemical reactivity descriptors were calculated using DFT. These are very important physical parameters to understand chemical and biological activities of the phytochemical constituents. The calculated HOMO-LUMO orbital energies can be used to estimate the ionization energy [10], electron affinity [10], electronegativity [11], electronic chemical potential [11], molecular hardness [12], molecular softness [12], and electrophilicity index [13] using the following equations:

$$
\begin{aligned}
& \text { ionization energy }(\mathrm{IE})=-\varepsilon_{\mathrm{HOMO}}, \\
& \text { electron affinity }(\mathrm{EA})=-\varepsilon_{\mathrm{LUMO}}, \\
& \text { electronegativity }(\chi)=(\mathrm{IE}+\mathrm{EA}) / 2, \\
& \text { electronic chemical potential }(\mu)=-\chi \text {, } \\
& \text { chemical hardness }(\eta)=(\mathrm{IE}-\mathrm{EA}) / 2, \\
& \text { chemical softness }(\sigma)=1 / \eta, \\
& \text { electrophilicity index }(\omega)=\mu^{2} / 2 \eta .
\end{aligned}
$$

\section{Results and Discussion}

DFT has long been used to predict certain functions such as ionization potentials [14], electron affinities [14-16], orbital energies [17], and molecular structures [18]. The frontier orbitals (HOMO and LUMO) of a chemical species are very important in defining its reactivity. Higher value of $\mathrm{HOMO}$ of a molecule has a tendency to donate electrons to appropriate acceptor molecule with low energy, empty molecular orbitals. $E_{\mathrm{LUMO}}$, on the other hand, indicates the ability of the molecule to accept electrons. The binding ability of the molecule increases with increasing HOMO and decreasing LUMO energy values. Thus, the lower the value of $E_{\text {LUMO }}$ is, the most probable the molecule would accept electrons. When the HOMO-LUMO energy gap $(\Delta E)$ decreases, the reactivity of the molecule increases leading to a decrease in the stability of the molecule. HOMO-LUMO gap is generally the lowest energy electronic excitation that is possible in a molecule.

Chemical hardness is associated with the stability and reactivity of a chemical system. The larger the HOMO-LUMO energy gap, the harder and more stable and less reactive the molecule. The smaller the HOMO-LUMO energy gap, the softer and less stable and more reactive the molecule. Electronic chemical potential is the negative value of electronegativity of a molecule. The greater the electronic chemical potential, the less stable or more reactive, the molecule will be. Electrophilicity index $(\omega)$ [13], measures the propensity or capacity of a species to accept electrons $[19,20]$. It is a measure of the stabilization in energy after a system accepts additional amount of electronic charge from the environment [21]. More stable molecule corresponds to lesser electrophilicity.

The results of density functional theory of phytochemical constituents identified from the ethanolic extracts of the fruits of Cucumis trigonus and Cucumis sativus are presented in Tables 1 and 2, respectively. By DFT studies, the presence of maximum number of phytochemical constituents in the ethanolic extract of the fruits of Cucumis trigonus showed more stability and biological activity than the ethanolic extract of the fruits of Cucumis sativus. The values of LUMOHOMO energy gap reflect the chemical activity of the molecule. The larger the HOMO-LUMO energy gap, the harder and more stable and less reactive the molecule [22, 23]. The decrease in the HOMO and LUMO energy gap explains the eventual charge transfer interaction taking place within the molecule due to the strong electron-accepting ability of the electron acceptor group. The strong charge transfer interaction is responsible for the bioactivity of the molecule.

Among the phytochemical constituents isolated and identified from the ethanolic extract of the fruits of Cucumis trigonus, glycodeoxycholic acid (A) showed more stability and biological activity as it shows less energy gap, low hardness, and more softness when compared with the standard silymarin (C). The energy gap, hardness, and softness of glycodeoxycholic acid are 0.0249, 0.0125, and 80.3213, respectively.

In the case of the phytochemical constituents isolated and identified from the ethanolic extract of the fruits of Cucumis sativus, 2-(2-methylcyclohexylidene)-hydrazinecarboxamide (B) shows less energy gap (2.4747), low hardness 


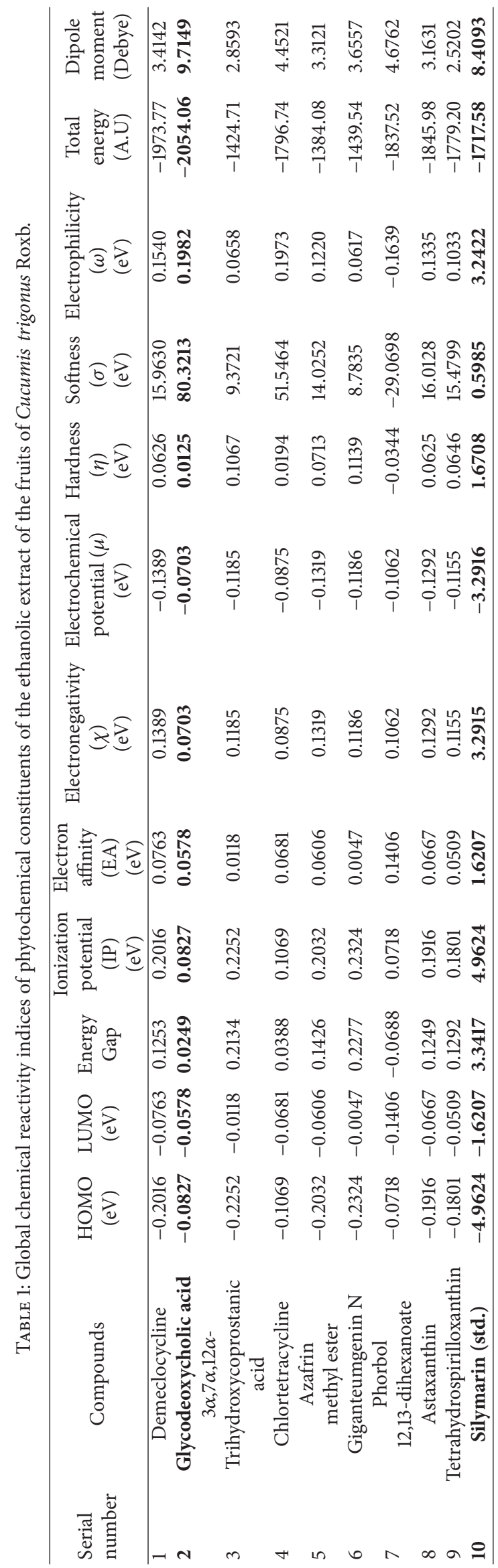




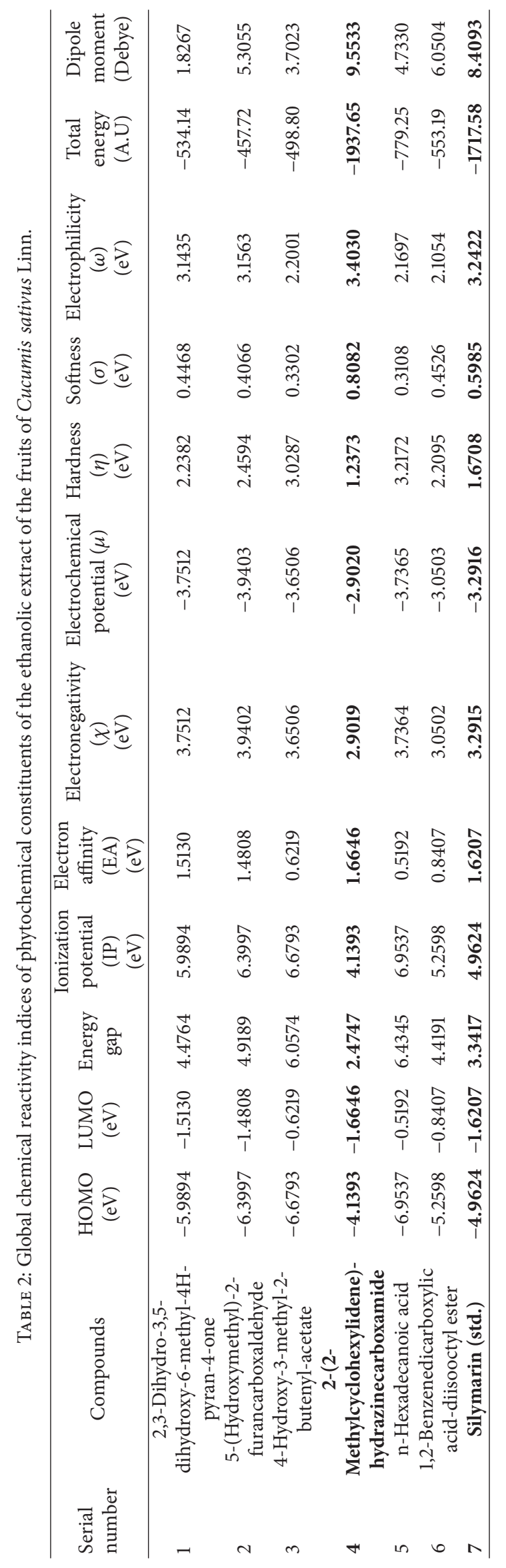




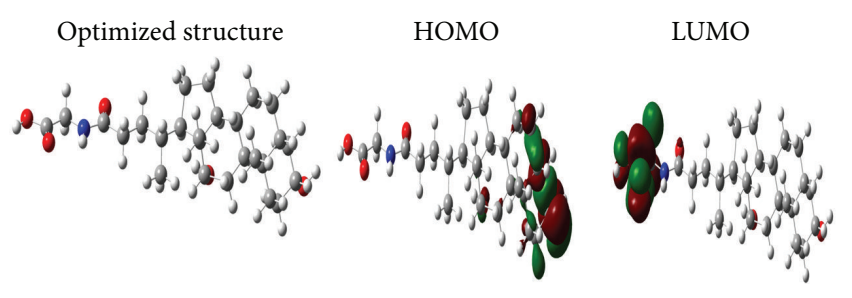

(A) Glycodeoxycholic acid

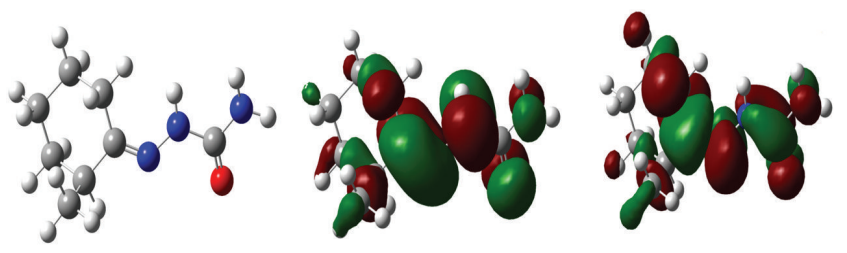

(B) 2-(2-Methylcyclohexylidene)-hydrazinecarboxamide

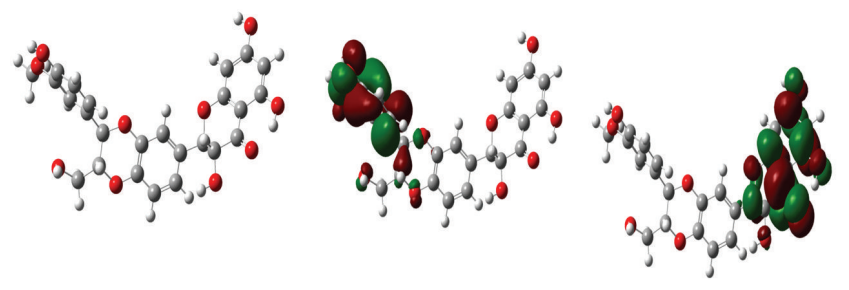

(C) Silymarin (standard drug)

FIGURE 1: DFT optimized geometries and frontier molecular orbitals of compounds (A), (B), and (C).

(1.2373), and more softness (0.8082) when compared with those of standard silymarin (C). DFT optimized geometries and frontier molecular orbitals of phytochemical constituents using B3LYP/6-311++G(d,p) basis set are presented in Figure 1.

From the theoretical study, it has been found that the biological activities of the phytochemical constituents isolated and identified from the ethanolic extract of the fruits of Cucumis trigonus were found to be more than that of the phytochemical constituents isolated and identified from the ethanolic extract of the fruits of Cucumis sativus. The activities were found to be more than that of the standard drug, silymarin. This is supported by the experimental studies carried out by our group earlier [24-26].

Administration of paracetamol to rats by oral route caused liver damage as indicated by a significant increase in serum enzymes. Treatment with the ethanolic extracts of the fruits of Cucumis trigonus and Cucumis sativus decreased the levels of elevated serum enzymes like serum glutamic oxalacetic transaminase (SGOT), serum glutamic pyruvate transaminase (SGPT), serum alkaline phosphatase (SALP), $\gamma$-glutamate transpeptidase (GGTP), total bilirubin, conjugated bilirubin, and unconjugated bilirubin and increased the levels of total protein, albumin, and globulin. In $500 \mathrm{mg} / \mathrm{kg}$ body weight dose of the ethanolic extract of the fruits of Cucumis trigonus treated groups, the levels of SGOT, SGPT, SALP, GGTP, total bilirubin, conjugated bilirubin, unconjugated bilirubin, total protein, albumin, and globulin were considerably reduced even lower than that of the Cucumis sativus treated groups and normal rats. The lowering of enzyme level is a definite indication of hepatoprotective action of the drug. The results indicate that the $500 \mathrm{mg} / \mathrm{kg}$ body weight of the ethanolic extract of the fruits of Cucumis trigonus possesses more hepatoprotective activity than the standard drug, silymarin.

The antimicrobial effects of Cucumis trigonus and Cucumis sativus fruit extracts were tested against the different strains. In Cucumis trigonus, the benzene extract was effective in the gram-negative bacteria and fungi. The ethanol extract showed the maximum activity against all the tested strains followed by water extract. In Cucumis sativus, there was no significant antibacterial activity of the pet ether, benzene, and chloroform extracts against the test organisms. The ethanol extract and water extract of the fruits of Cucumis sativus only possess significant inhibitory activities against all the grampositive, gram-negative bacteria and fungi. The difference in the activity may be due to the different secondary metabolites present in the various extracts. From these results it may be concluded that the fruits of Cucumis trigonus showed better activity than the Cucumis sativus.

The biological activities such as hepatoprotective activity $[24,25]$ and antimicrobial activity [26] of phytochemical constituents identified from the ethanolic extract of the fruits of Cucumis trigonus were more than that of the phytochemical constituents identified from the ethanolic extract of the fruits of Cucumis sativus.

\section{Conclusion}

Chemical reactivity descriptors such as HOMO-LUMO energy cap, softness, total energy, chemical hardness, electronic chemical potential, and electrophilicity of bioactive compounds have been examined theoretically. From the results it may be concluded that phytochemical constituents of the fruits of Cucumis trigonus and Cucumis sativus exhibited more biological activity when compared to that of the standard drug, silymarin. Based on the analysis of geometries, electronic structures, the biological activity of phytochemical constituents of the ethanolic extract of the fruits of Cucumis trigonus was found to be higher than that of phytochemical constituents of the ethanolic extract of the fruits of Cucumis sativus. The results indicate that the density functional theory is a valuable tool for predicting biological activity of phytochemical constituents. The HOMO-LUMO energy gap reveals the stability index of compounds.

\section{Conflict of Interests}

The authors declare that there is no conflict of interests regarding the publication of this paper.

\section{References}

[1] K. Fukui, T. Yonezawa, and H. Shingu, "A molecular orbital theory of reactivity in aromatic hydrocarbons," Journal of Chemical Physics, vol. 20, no. 4, pp. 722-725, 1952. 
[2] L. H. Mendoza-Huizar and C. H. Rios-Reyes, "Chemical reactivity of Atrazine employing the Fukui function," Journal of the Mexican Chemical Society, vol. 55, no. 3, pp. 142-147, 2011.

[3] J. Hafner, "Materials simulations using VASP-a quantum perspective to materials science," Computer Physics Communications, vol. 177, pp. 6-13, 2007.

[4] S. Gopalakrishnan and T. Kalaiarasi, "Identification of chemical compounds from the fruits of Cucumis trigonus Roxb. by GCMS analysis," International Journal of Phytopharmacy, vol. 2, pp. 122-128, 2012.

[5] S. Gopalakrishnan and T. Kalaiarasi, "Hepatoprotective activity of the fruits of Cucumis sativus Linn," International Journal of Pharmaceutical Sciences Review and Research, vol. 20, no. 2, pp. 229-234, 2013.

[6] M. J. Frisch, G. W. Trucks, H. B. Schlegel et al., Gaussian 09, Revision A.01, Gaussian, Wallingford, Conn, USA, 2009.

[7] A. D. Becke, "Density-functional thermochemistry. II. The effect of the Perdew-Wang generalized-gradient correlation correction," The Journal of Chemical Physics, vol. 97, no. 12, pp. 9173-9177, 1992.

[8] A. D. Becke, "Density-functional thermochemistry. III. The role of exact exchange," The Journal of Chemical Physics, vol. 98, no. 7, pp. 5648-5652, 1993.

[9] C. Lee, W. Yang, and R. G. Parr, "Development of the ColleSalvetti correlation-energy formula into a functional of the electron density," Physical Review B: Condensed Matter, vol. 37, no. 2, pp. 785-789, 1988.

[10] J. B. Foresman and A. Frisch, Exploring Chemistry with Electronic Structure Methods, Gaussian, Pittsburg, Pa, USA, 1995.

[11] L. Pauling, The Nature of the Chemical Bond, Cornell University Press, Ithaca, NY, USA, 1960.

[12] P. Senet, "Chemical hardnesses of atoms and molecules from frontier orbitals," Chemical Physics Letters, vol. 275, no. 5-6, pp. 527-532, 1997.

[13] R. G. Parr and R. G. Pearson, "Absolute hardness: companion parameter to absolute electronegativity," Journal of the American Chemical Society, vol. 105, no. 26, pp. 7512-7516, 1983.

[14] C. Zhan, J. A. Nichols, and D. A. Dixon, "Ionization potential, electron affinity, electronegativity, hardness, and electron excitation energy: Molecular properties from density functional theory orbital energies," The Journal of Physical Chemistry A, vol. 107, no. 20, pp. 4184-4195, 2003.

[15] J. C. Rienstra-Kiracofe, G. S. Tschumper, H. F. Schaefer III, S. Nandi, and G. B. Ellison, "Atomic and molecular electron affinities: photoelectron experiments and theoretical computations," Chemical Reviews, vol. 102, no. 1, pp. 231-282, 2002.

[16] J. C. Rienstra-Kiracofe, C. J. Barden, S. T. Brown, and H. F. Schaefer III, "Electron affinities of polycyclic aromatic hydrocarbons," The Journal of Physical Chemistry A, vol. 105, no. 3, pp. 524-528, 2001.

[17] J. Muscat, A. Wander, and N. M. Harrison, "On the prediction of band gaps from hybrid functional theory," Chemical Physics Letters, vol. 342, no. 3, pp. 397-401, 2001.

[18] L. A. Curtiss, P. C. Redfern, K. Raghavachari, and J. A. Pople, "Gaussian-3X (G3X) theory: use of improved geometries, zeropoint energies, and Hartree-Fock basis sets," Journal of Chemical Physics, vol. 114, no. 1, pp. 108-117, 2001.

[19] A. Vektariene, G. Vektaris, and J. Svoboda, "A theoretical approach to the nucleophilic behavior of benzofused thieno[3, 2-b]furans using DFT and HF based reactivity descriptors," Arkivoc, vol. 2009, no. 7, pp. 311-329, 2009.
[20] R. G. Parr, L. V. Szentpály, and S. Liu, "Electrophilicity index," Journal of the American Chemical Society, vol. 121, no. 9, pp. 1922-1924, 1999.

[21] S. Liu, "Dynamic behavior of chemical reactivity indices in density functional theory: a Bohn-Oppenheimer quantum molecular dynamics study," Journal of Chemical Sciences, vol. 117, no. 5, pp. 477-483, 2005.

[22] P. K. Chattaraj and B. Maiti, "HSAB principle applied to the time evolution of chemical reactions," Journal of the American Chemical Society, vol. 125, no. 9, pp. 2705-2710, 2003.

[23] K. D. Sen and D. M. P. Mingos, Chemical Hardness: Structure and Bonding, Springer, Berlin, Germany, 1993.

[24] S. Gopalakrishnan and T. Kalaiarasi, "Hepatoprotective activity of the ethanolic extract of the fruits of Cucumis trigonus Roxb," International Journal of Pharmacy and Pharmaceutical Sciences, vol. 5, no. 2, pp. 268-272, 2013.

[25] S. Gopalakrishnan and T. Kalaiarasi, "Hepatoprotective activity of the fruits of Cucumis sativus (L.)," International Journal of Pharmaceutical Sciences Review and Research, vol. 20, no. 2, pp. 229-234, 2013.

[26] S. Gopalakrishnan, T. Kalaiarasi, and R. Rajameena, "Evaluation of antimicrobial activity of the fruits of Cucumis trigonus Roxb," Research Journal of Pharmacy, vol. 3, no. 5, pp. 256-258, 2012. 

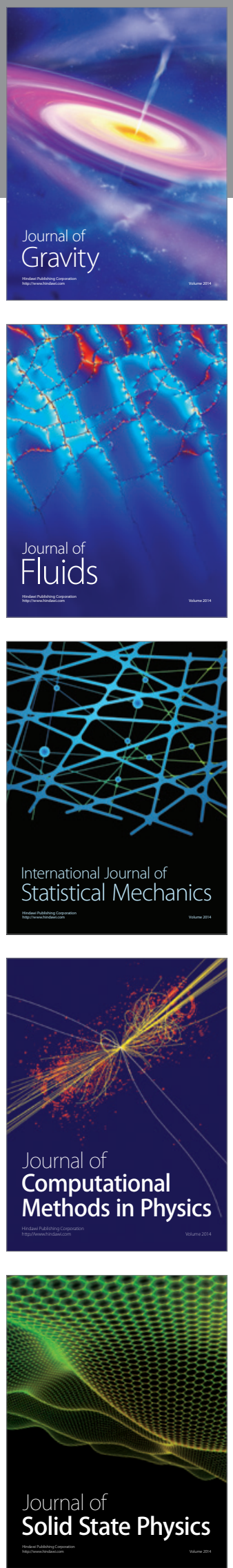

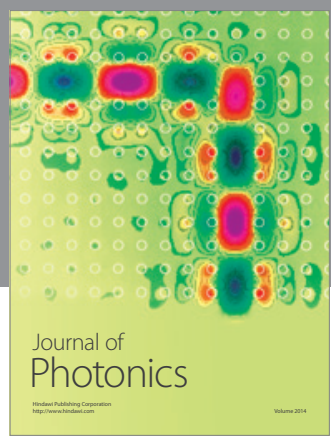

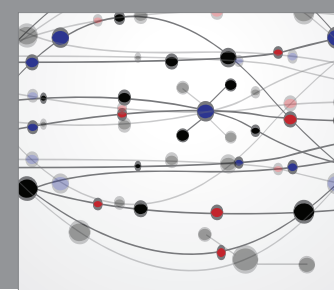

The Scientific World Journal

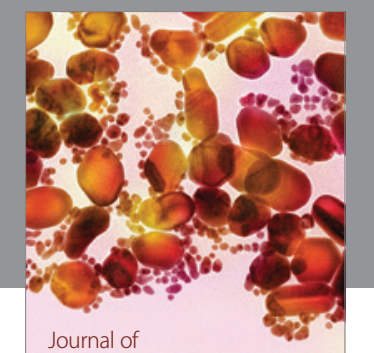

Soft Matter
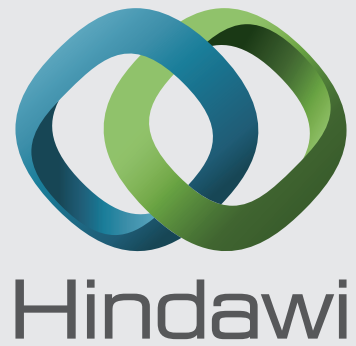

Submit your manuscripts at

http://www.hindawi.com
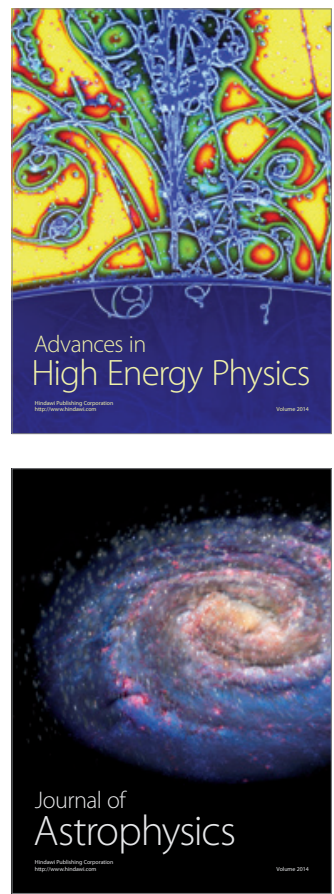
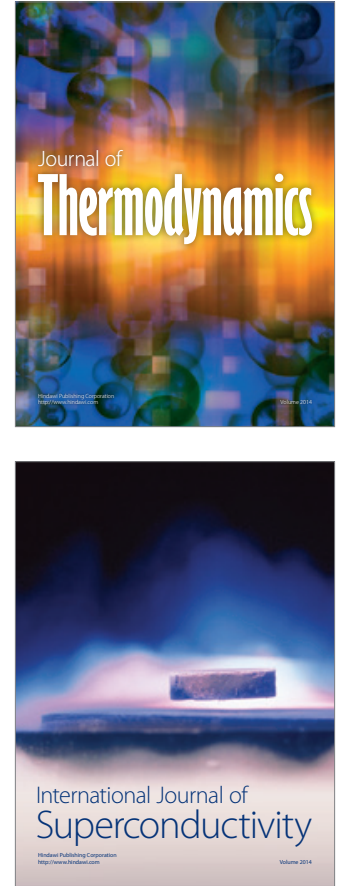
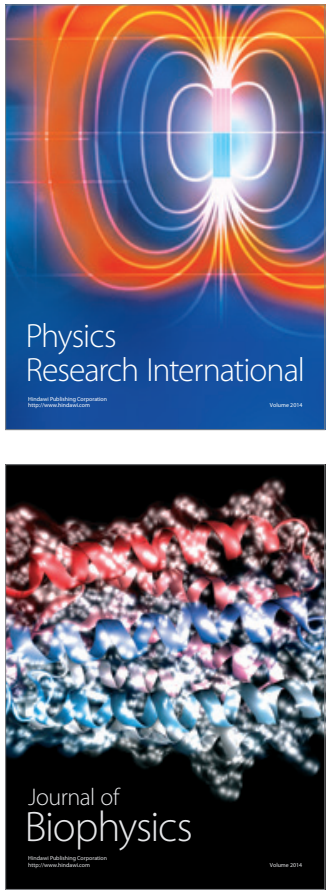
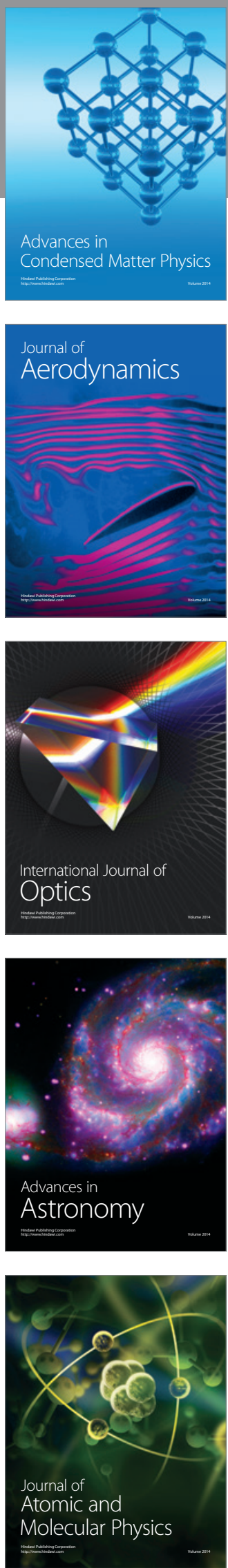